\title{
OPTICAL IDENTIFICATION OF FOUR HARD X-RAY SOURCES FROM THE SWIFT ALL-SKY SURVEY
}

\author{
(c) 2021 A. A. Lutovinov ${ }^{* 1}$, R. A. Burenin ${ }^{1}$, M. G. Revnivtsev ${ }^{1}$, S. Yu. Sazonov ${ }^{1}$, O. N. Sholukhova ${ }^{2}$, \\ and A.F. Valeev ${ }^{2}$ \\ (1) Space Research Institute, Russian Academy of Sciences, Moscow, Russia \\ (2) Special Astrophysical Observatory, Russian Academy of Sciences, Nizhnii Arkhyz, Russia \\ Received 24 December 2011
}

\begin{abstract}
We present the results of our optical identifications of four hard X-ray sources from the Swift all-sky survey. We obtained optical spectra for each of the program objects with the 6-m BTA telescope (Special Astrophysical Observatory, Russian Academy of Sciences, Nizhnii Arkhyz), which allowed their nature to be established. Two sources ( SWIFT J2237.2+6324 and SWIFT J2341.0+7645) are shown to belong to the class of cataclysmic variables (suspected polars or intermediate polars).

The measured emission line width turns out to be fairly large $(F W H M \sim 15-25 \AA)$, suggesting the presence of extended, rapidly rotating $(v \simeq 400-600 \mathrm{~km} / \mathrm{s})$ accretion disks in the systems. Apart from line broadening, we have detected a change in the positions of the line centroids for SWIFT J2341.0+7645, which is most likely attributable to the orbital motion of the white dwarf in the binary system. The other two program objects (SWIFT J0003.3+2737 and SWIFT J0113.8+2515) are extragalactic in origin: the first is a Seyfert 2 galaxy and the second is a blazar at redshift $z=1.594$. Apart from the optical spectra, we provide the X-ray spectra for all sources in the $0.6-10 \mathrm{keV}$ energy band obtained from XRT/Swift data.
\end{abstract}

Keywords: X-ray sources, cataclysmic variables, white dwarfs, active galactic nuclei

\section{INTRODUCTION}

The all-sky surveys currently conducted in the hard (> $15 \mathrm{keV}$ ) X-ray energy band by the INTEGRAL (Winkler et al. 2003) and Swift '(Gehrels et al. 2004) observatories are widely used to solve various problems: discovering new sources (see, e.g., Krivonos et al. 2007, 2010; Bird et al. 2010; Baumgartner et al. 2010; Cusumano et al. 2010), investigating the physical properties of various objects (see, e.g., Filippova et al. 2005; Barlow et al. 2006; Lutovinov and Tsygankov 2009), investigating the statistical properties of objects of various classes (see, e.g., Sazonov et al. 2007, 2008; Lutovinov et al. 2005; Revnivtsev et al. 2008, etc). The key point for a successful solution of such problems is to establish the nature of the program objects; the higher the completeness of the identifications, the more valuable a particular survey and the wider the range of problems it can solve.

To determine the nature of detected hard X-

*e-mail: aal@iki.rssi.ru ray sources, various groups, including our group, perform additional observations with X-ray, optical, and infrared telescopes (see, e.g. Sazonov et al. 2005; Bikmaev et al. 2006, 2008; Masetti et al. 2007, 2010; Burenin et al. 2008, 2009; Tomsick et al. 2009; Lutovinov et al. 2010, 2012).

In this paper, we determine the nature of four sources from the Swift all-sky survey based on their $\mathrm{X}$-ray and optical properties. This work is the next one in the program of identifying hard X-ray sources that we carry out with the BTA and RTT-150 telescopes.

\section{OBSERVATIONS AND DATA REDUCTION}

For our optical observations, we selected four hard X-ray sources (SWIFT J0003.3+2737, SWIFT J0113.8+2515, SWIFT J2237.2+6324 and SWIFT J2341.0+7645; see the table) from the 58month Swift catalog (Baumgartner et al. 2010). All these objects were detected with the BAT/Swift telescope at low flux and confidence $(\sim 5 \sigma)$ levels, which leads to large errors in the localization of such objects $\left(\sim 6-7^{\prime}\right.$, see Tueller et al. 
Table 1. The list of hard X-ray sources

\begin{tabular}{lcc}
\hline \hline Name & $\begin{array}{c}\text { RA } \\
(\mathrm{J} 2000)\end{array}$ & $\begin{array}{c}\text { Dec } \\
(\mathrm{J} 2000)\end{array}$ \\
\hline SWIFT J0003.3+2737 & $00^{h} 03^{m} 20.2 s$ & $27^{\circ} 37^{\prime} 23^{\prime \prime}$ \\
SWIFT J0113.8+2515 & $01^{h} 13^{m} 48.7 s$ & $25^{\circ} 14^{\prime} 38^{\prime \prime}$ \\
SWIFT J2237.2+6324 & $22^{h} 36^{m} 26.2 s$ & $63^{\circ} 27^{\prime} 50^{\prime \prime}$ \\
SWIFT J2341.0+7645 & $23^{h} 40^{m} 41.8 s$ & $76^{\circ} 44^{\prime} 31^{\prime \prime}$ \\
\hline
\end{tabular}

2010). In spite of this, subsequent observations with the Swift telescope allowed one to identify them in the soft X-ray energy band $(0.6-10$ $\mathrm{keV}$ ) and to improve significantly the localization accuracy (to several arcseconds). The latter allowed us to unambiguously determine the optical object associated with the hard X-ray source.

Our optical observations were performed on November $4 / 5,2011$, with the SCORPIO spectrometer (Afanasiev and Moiseev 2005) attached to the 6-m BTA telescope (Special Astrophysical Observatory, Russian Academy of Sciences). We obtained two spectra, each with an exposure time of $600 \mathrm{~s}$, for SWIFT J0003.3+2737; for each of the remaining sources, we took two spectra, each with an exposure time of $900 \mathrm{~s}$. Here, we present the total spectra for each source, except SWIFT J2341.0+7645 for which we detected a shift in the spectra due to the orbital motion of the object in the binary system (for more details, see below). During these observations, we used the 3D holographic grating VPHG550G that provided the highest light efficiency and a wide spectral range $(3700-7800 \AA)$. The spectral resolution was $\approx 6 \AA$ (FWHM).

We reduced the optical data in a standard way using the IRAF1 software package 1 and our own software. It should be noted that we failed to observe the spectrophotometric standards because of technical problems. Therefore, below all spectra are presented in relative units and are normalized to the continuum. The X-ray spectra of the sources in the $0.6-10 \mathrm{keV}$ energy band were obtained from $\mathrm{XRT} /$ Swift data. They were reduced and analyzed using appropriate software 2 and the FTOOLS 6.7 software package.

\section{RESULTS}

SWIFT J0003.3 +2737 . The sky field around SWIFT J0003.3+2727 was observed with the

\footnotetext{
${ }^{1}$ http://iraf.noao.edu

${ }^{2}$ http://swift.gsfc.nasa.gov
}

XRT/Swift telescope on January 21, 2011, with a total exposure time of $\simeq 8.4 \mathrm{ks}$. Three rather faint objects are detected in the BAT error circle of the source; one of them is approximately twice as intense as the other two in the $0.6-10 \mathrm{keV}$ energy band. Moreover, the last two sources essentially disappear in the image of the sky field obtained in the $6-10 \mathrm{keV}$ energy band, while the first object with (J2000) coordinates $\mathrm{RA}=00^{h} 03^{m} 27.4^{s}$, Dec $=27^{\circ} 39^{\prime} 18^{\prime \prime}$ (the localization accuracy is $\sim 5^{\prime \prime}$ ) is still detected with confidence. Thus, we may conclude that precisely this object is the soft X-ray counterpart of SWIFT J0003.3+2727.

Comparison of the XRT image with the SDSS images shows that SWIFT J0003.3+2727 can be unambiguously identified in the optical band with a relatively bright object, $m_{r}=16.3$ (Fig [a). Its optical spectrum clearly shows Balmer emission lines, narrow NII lines, the [OIII] 4959,5007 lines, and others (Fig,10), typical of a Seyfert 2 galaxy at redshift $z=0.03946 \pm 0.00002$.

The X-ray spectrum of SWIFT J0003.3+2727 shown in Fig 10 can formally be well fitted by a simple power-law dependence of the photon flux density on energy $d N / d E \propto E^{-\Gamma}$ with a photon index $\Gamma \simeq-1$. However, such a photon index is atypical of the spectra of active galactic nuclei (AGNs), that most likely connected with a significant absorption in its spectrum. Unfortunately, the low quality of the spectrum does not allow this question to be investigated in detail. We can only fix the photon index at $\Gamma=1.7$, typical of AGNs and estimate it as $N_{H} \simeq 10^{23} \mathrm{~cm}^{-2}$ by adding absorption to the model.

The X-ray flux from the source in the $2-10 \mathrm{keV}$ energy band is $F_{X} \simeq 1.5 \times 10^{-12} \mathrm{erg} \mathrm{cm}^{-2} \mathrm{~s}^{-1}$, which corresponds to a luminosity $L_{X} \simeq 5 \times 10^{42}$ $\operatorname{erg~s}^{-1}$ for the measured $z$.

SWIFT J0113.8+2515. The source SWIFT J0113.8+2515 was observed with the XRT telescope three times with a time difference of about one year (February 28, 2010; March 1 and 2, 2011), the total exposure time being $\simeq 8 \mathrm{ks}$. Since we found no significant changes in the detected flux and the shape of its spectrum, we analyzed the total $\mathrm{X}$-ray emission from the program object.

As we see from Fig 2 a, one object with $m_{r}=19.1$ (from SDSS data) falls into the XRT error circle of the source $\left(\mathrm{RA}=01^{h} 13^{m} 22.7^{s}\right.$, Dec $=25^{\circ} 18^{\prime} 54^{\prime \prime}$, $\mathrm{J} 2000$, the localization accuracy is $\left.\sim 4^{\prime \prime}\right)$. The optical spectrum of this object taken with the BTA telescope reveals two relatively weak emission lines (Fig 2b). We identified these lines with the broad 

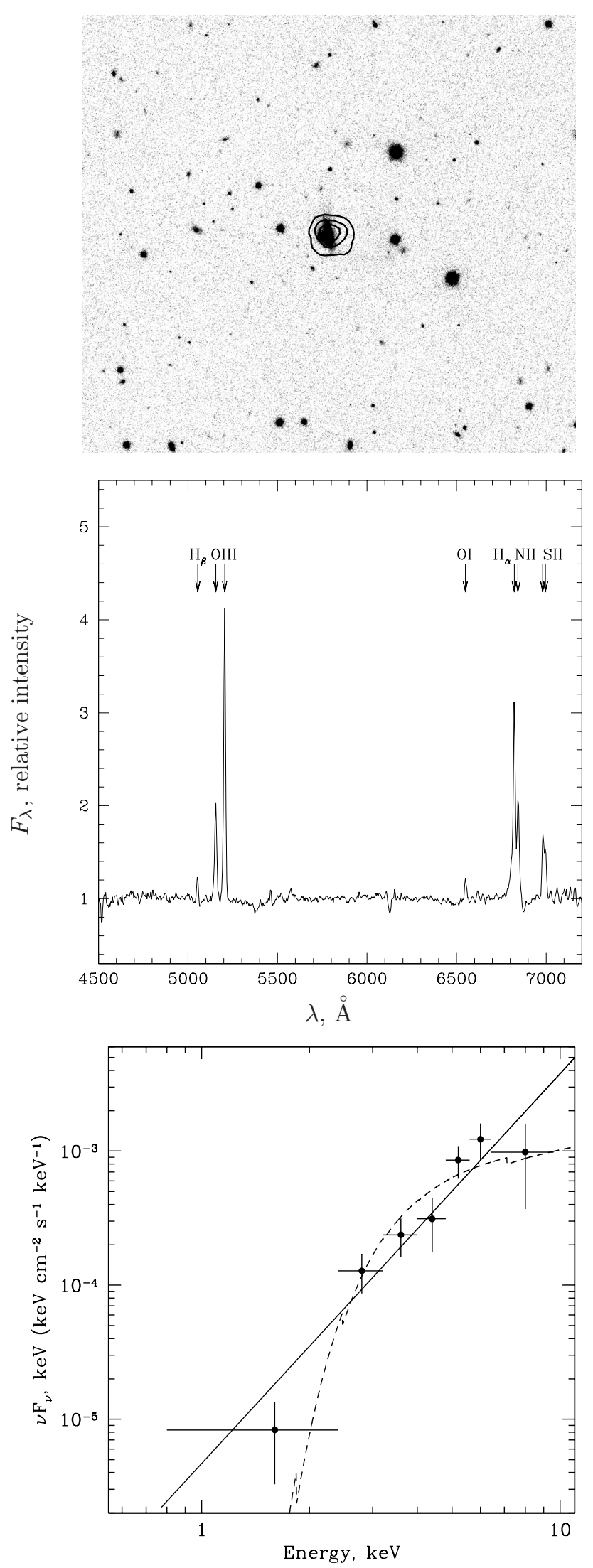

Fig. 1. (a) SDSS image of the sky field with SWIFT J0003.3+2727; the contours indicate the soft X-ray intensity levels from XRT data. (b) The optical spectrum of SWIFT J0003.3+2727 taken with the BTA telescope. The main emission lines are labeled. (c) The energy spectrum of SWIFT J0003.3+2727 from XRT data: the solid and dashed lines indicate, respectively, the best fits by a power law and a power law with a slope of 1.7 and absorption. 

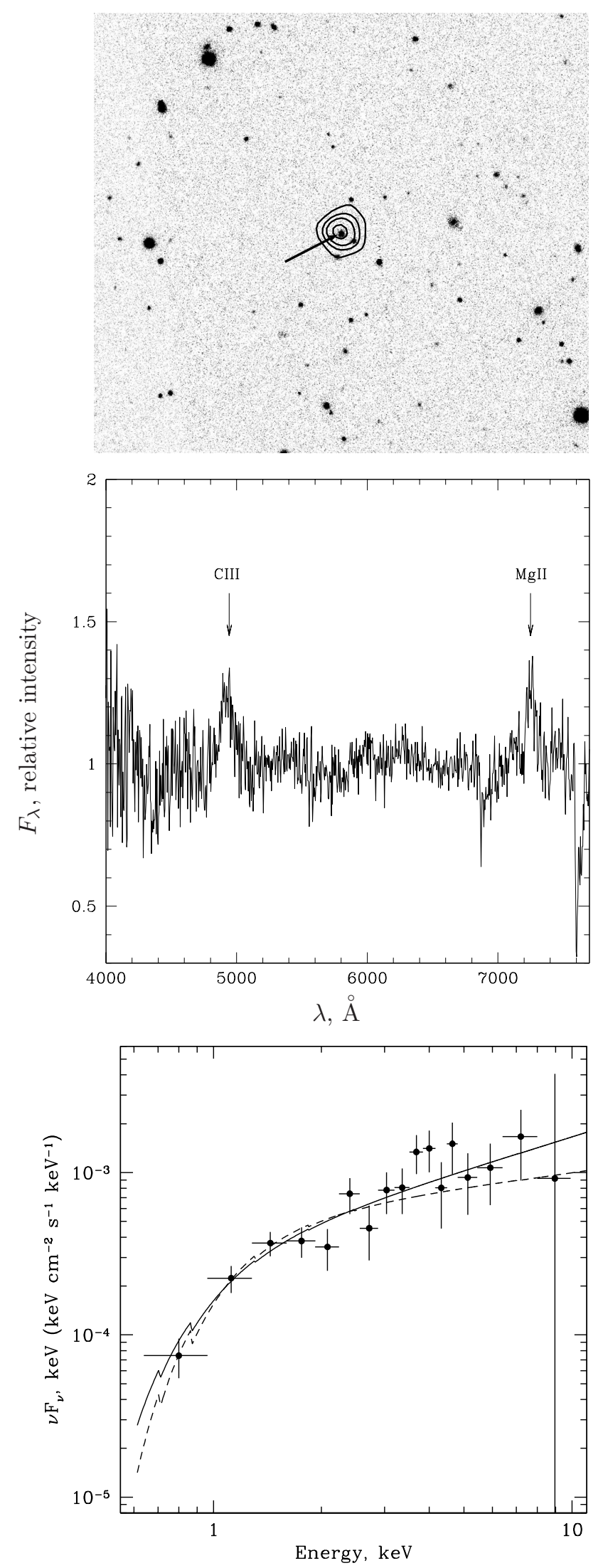

Fig. 2. (a) Sky field around SWIFT J0113.8+2515 from SDSS data; the contours indicate the soft X-ray intensity levels from XRT data, the arrow indicates of the optical counterpart. (b) The optical spectrum of $S W I F T$ $J 0113.8+2515$ taken with the BTA telescope. The two emission lines we managed to identify are labeled. (c) The energy spectrum of SWIFT J0113.8+2515 from XRT data: the solid and dashed lines indicate, respectively, the best fits (see the text) by a power law and a power law with a slope of 1.7 and absorption. 


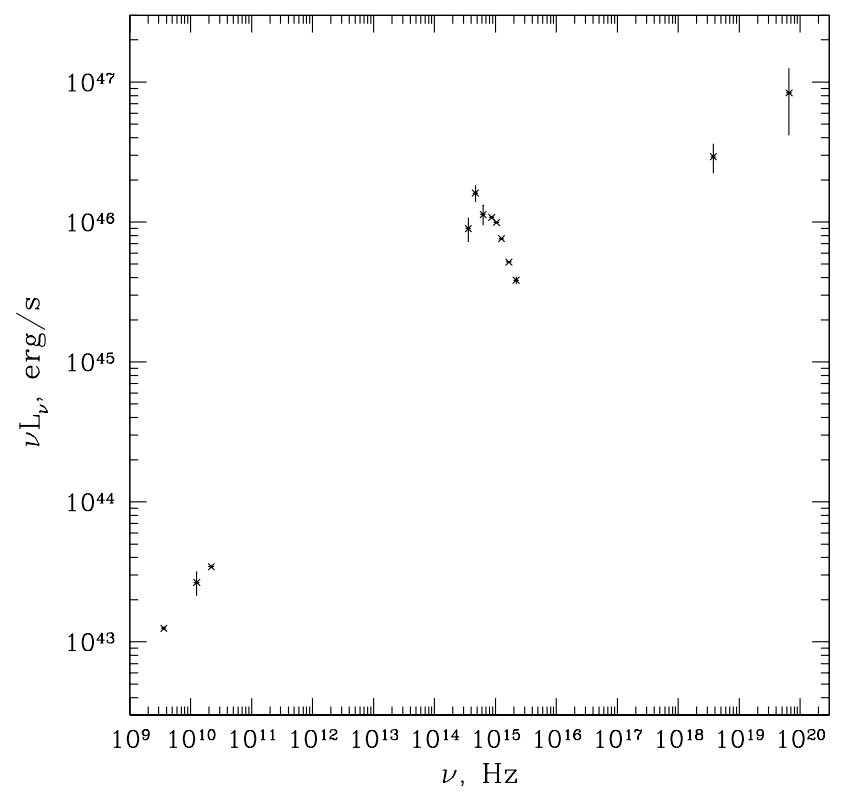

Fig. 3. Spectral energy distribution for SWIFT $J 0113.8+2515$ based on data from different radio telescopes, the infrared 2MASS survey, the optical SDSS survey, the XRT telescope, and the hard X-ray BAT instrument (both onboard the SWIFT observatory). The infrared and optical measurements were corrected for Galactic extinction with $E(B-V)=0.080$ (Schlegel et al. 1998).

CIII $1909 \AA$ and MgII $2798 \AA$ lines with a Doppler width of $\sim 5000 \mathrm{~km} / \mathrm{s}(F W H M)$ at redshift $z=$ $1.594 \pm 0.002$. Thus, our measurements indicate that $S$ WIFT J0113.8+2515 is a quasar.

Our search in astronomical catalogs showed that apart from the optical band, the object was previously detected in the near infrared (in the 2MASS twomicron survey) and with several radio telescopes. In addition, it was detected at a low confidence level in the ROSAT all-sky X-ray survey (the source $R X$ J0113.2+2518; Brinkmann et al. 1997). Figure 3 shows the source's spectral energy distribution from the available data. We calculated the luminosities using the cosmological model with $\Omega_{\mathrm{m}}=0.3, \Omega_{\Lambda}=0.7$ and $H_{0}=$ $72 \mathrm{~km} / \mathrm{s} / \mathrm{Mpc}$. The flat (with a spectral slope $\alpha \sim 0$ ) radio spectrum and the presence of two emission peaks (in the optical band and in the hard $\mathrm{X}$-ray or harder energy band) indicate that the object is a flat-spectrum radio quasar, i.e. a blazar. The derived spectral energy distribution is in satisfactory agreement with the well-known blazar sequence: the dependence of the positions of the two (synchrotron and Comptonization) emission peaks on the object's luminosity (e.g., Fossati et al. 1998).
The soft-X-ray spectrum of the source is well fitted by a simple power law, possibly modified by absorption (Fig 22). Assuming that the absorption occurs inside or near the source, i.e. at redshift $z=1.594$, the best values of the photon index and the column density of neutral matter turn out to be $\Gamma=1.10 \pm 0.20, N_{H}=2.3_{-1.0}^{+1.8} \times 10^{22} \mathrm{~cm}^{-2}$. The $\chi^{2}$ value per degree of freedom is $0.73(26)$. The derived upper limit for the absorbtion column density turns out to be considerably higher (even if the redshift is taken into account) than the column density of photoabsorbing matter on the line of sight in the Galaxy, $N_{H}=0.06 \times 10^{22} \mathrm{~cm}^{-2}$ (Dickey and Lockman 1990). The BAT measurement of the flux in the harder $14-195 \mathrm{keV}$ energy band confirms that the hard spectrum typical of blazars (flatspectrum radio quasars) extends at least to the range of soft gamma-ray energies. The power-law model with a fixed slope $\Gamma=1.7$ and absorption $N_{H}=(5 \pm 1) \times 10^{22} \mathrm{~cm}^{-2}$ at $z=1.594$ that could correspond to an ordinary quasar (not of the blazar type) describes the $\mathrm{X}$-ray spectrum slightly more poorly $\left(\chi^{2}=0.95\right.$ per degree of freedom $)$.

The flux from the source in the $2-10 \mathrm{keV}$ energy band, $F_{X} \simeq 3 \times 10^{-12} \mathrm{erg} \mathrm{cm}^{-2} \mathrm{~s}^{-1}$, corresponds to a luminosity $L_{X} \simeq 1.9 \times 10^{46}$ $\operatorname{erg~s}^{-1}$ (without a correction for the absorption). Thus, SWIFT J0113.8+2515 is one of the brightest blazars detected in the INTEGRAL and Swift allsky surveys.

SWIFT J2237.2 +6324 . Three soft X-ray sources were detected in the XRT error circle of SWIFT J2237.2+6324. The emission from two of them turns out to be considerably less intense than that from the third object with coordinates $\mathrm{RA}=22^{h} 36^{m} 37.3^{s}, \quad$ Dec $=63^{\circ} 29^{\prime} 31^{\prime \prime}(\mathrm{J} 2000)$ and a localization accuracy of $\sim 4^{\prime \prime}$. The latter circumstance makes this object the most probable source of the observed emission in the hard Xray energy band as well. The sky field containing SWIFT J2237.2+6324 was observed with the XRT telescope three times in 2008 (on July 9, 16, and 17) with a total exposure time of $\simeq 16 \mathrm{ks}$. During these observations, the flux from the program source changed by a factor of $\approx 2$. However, we found no ensuing significant changes in the shape of the spectrum, possibly because the quality of the latter was not very high, which, in turn, is related to the faintness of the object itself. Thus, all of the subsequent reasoning refers to the total spectrum obtained during the above observations.

Comparison of the soft X-ray images with the digitized Palomar Observatory Sky Survey (POSS) 

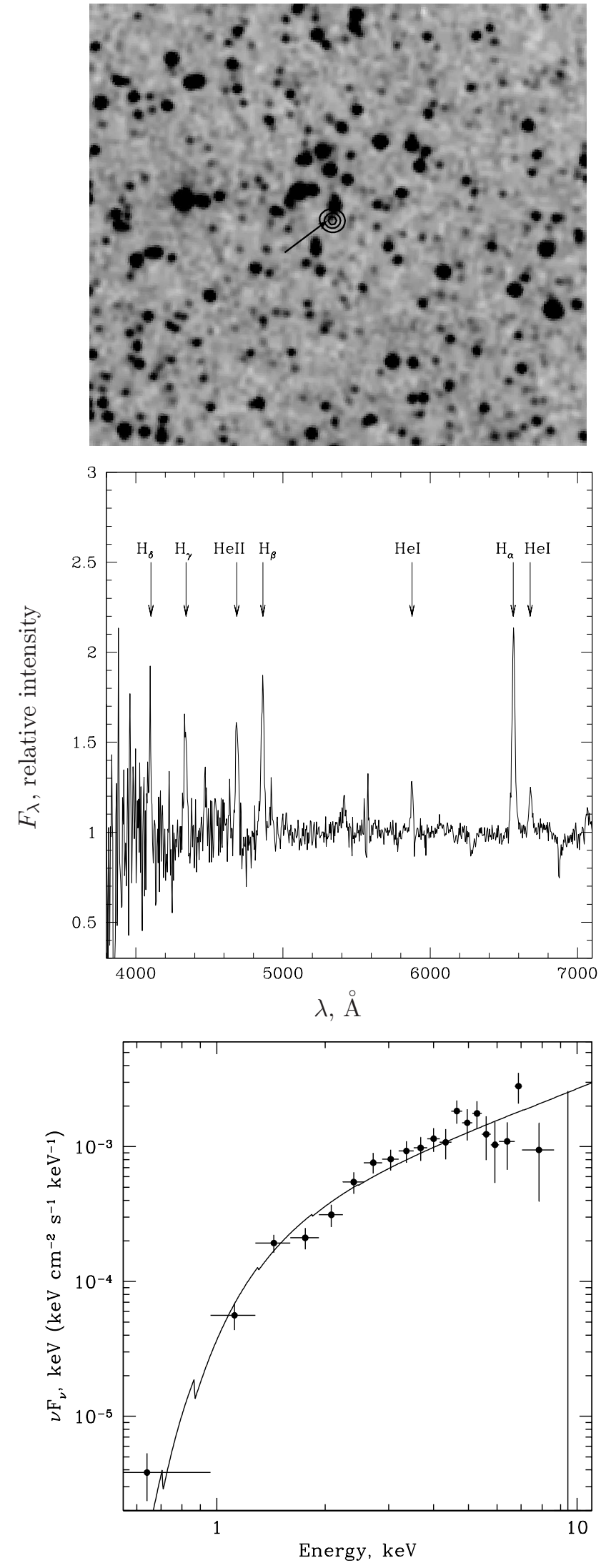

Fig. 4. (a) Sky field near SWIFT J2237.2+6324 from digitized POSS) plates; the contours indicate the soft Xray intensity levels from XRT data; the arrow indicates the position of the optical counterpart. (b) The optical spectrum of SWIFT J223\%.2+6324 taken with the BTA telescope. The main emission lines are labeled. (c) The energy spectrum of SWIFT J2237.2+6324 from XRT data: the solid line indicates the best fit. plates indicates that there is a bright optical object near the improved error circle of SWIFT J2237.2+6324, but it does not fall into this circle (see Fig 4 4 ). The most probable optical counterpart in the system is the fainter star with $m_{I} \simeq 18$ lying several arcseconds southwest of the brighter star (indicated by the arrow in Fig 4a). To establish its nature, we performed spectroscopic observations with the BTA telescope; during these observations, the slit was positioned in such a way that both objects fell into it simultaneously. The spectrum of the optical counterpart of SWIFT J2237.2+6324 is shown in Fig 4 b. It exhibits a set of bright hydrogen and helium emission lines characteristic of the spectra of accretion disks around white dwarfs (see, e.g., Williams and Ferguson 1982). Thus, this object is a cataclysmic variable, an accreting white dwarf in a binary system. It is important to note that the width of the lines detected in the $\operatorname{spectrum}(F W H M \simeq 15-20 \AA)$ exceeds considerably the spectral resolution of the grating used $(\approx 6 \AA$ ) , suggesting their internal broadening. Such a broadening can be due to the rotation of emitting matter in the accretion disk around the white dwarf. The velocities derived from the observed emission line widths $(v \simeq 300-450 \mathrm{~km} / \mathrm{s})$ are in agreement with the direct measurements in similar systems (e.g., Szkody et al. 2001).

The soft X-ray spectrum of SWIFT J2237.2+6324 can be fitted by a power-law dependence of the photon flux density on energy modified by photoabsorption with the following parameters (Fig 4c): $\Gamma=0.95 \pm 0.17$, $N_{H}=(0.77 \pm 0.17) \times 10^{22} \mathrm{~cm}^{-2}$, the flux in the $2-10 \mathrm{keV}$ energy band $F_{X}=3.5_{-0.8}^{+0.4} \times 10^{-12} \mathrm{erg}$ $\mathrm{cm}^{-2} \mathrm{~s}^{-1}$. Note that the derived absorption agrees well with the interstellar absorption toward the source, $N_{H}=0.76 \times 10^{22}$ atoms $\mathrm{cm}^{-2}$ (Dickey and Lockman 1990).

SWIFT J2341.0+7645. The XRT observations on July 31 and August 4, 2010, with a total exposure time of $\simeq 10.5 \mathrm{ks}$ allowed SWIFT J2341.0+7645 to be unambiguously identified with a soft Xray source with coordinates (J2000) $\mathrm{RA}=23^{h} 40^{m}$ $20.6^{s}, \mathrm{Dec}=76^{\circ} 42^{\prime} 09^{\prime \prime}$ and a localization accuracy of $\sim 3^{\prime \prime}$ (the latter coincides with the X-ray source 1RXS J234015.8+764207 from the ROSAT survey). Comparison of the X-ray and optical images shows that only one optical object with $m_{I} \simeq 17$ falls into this error circle (Fig $5 \mathrm{a}$ ).

The optical spectrum of the source exhibits a large set of hydrogen and helium lines (at redshift 

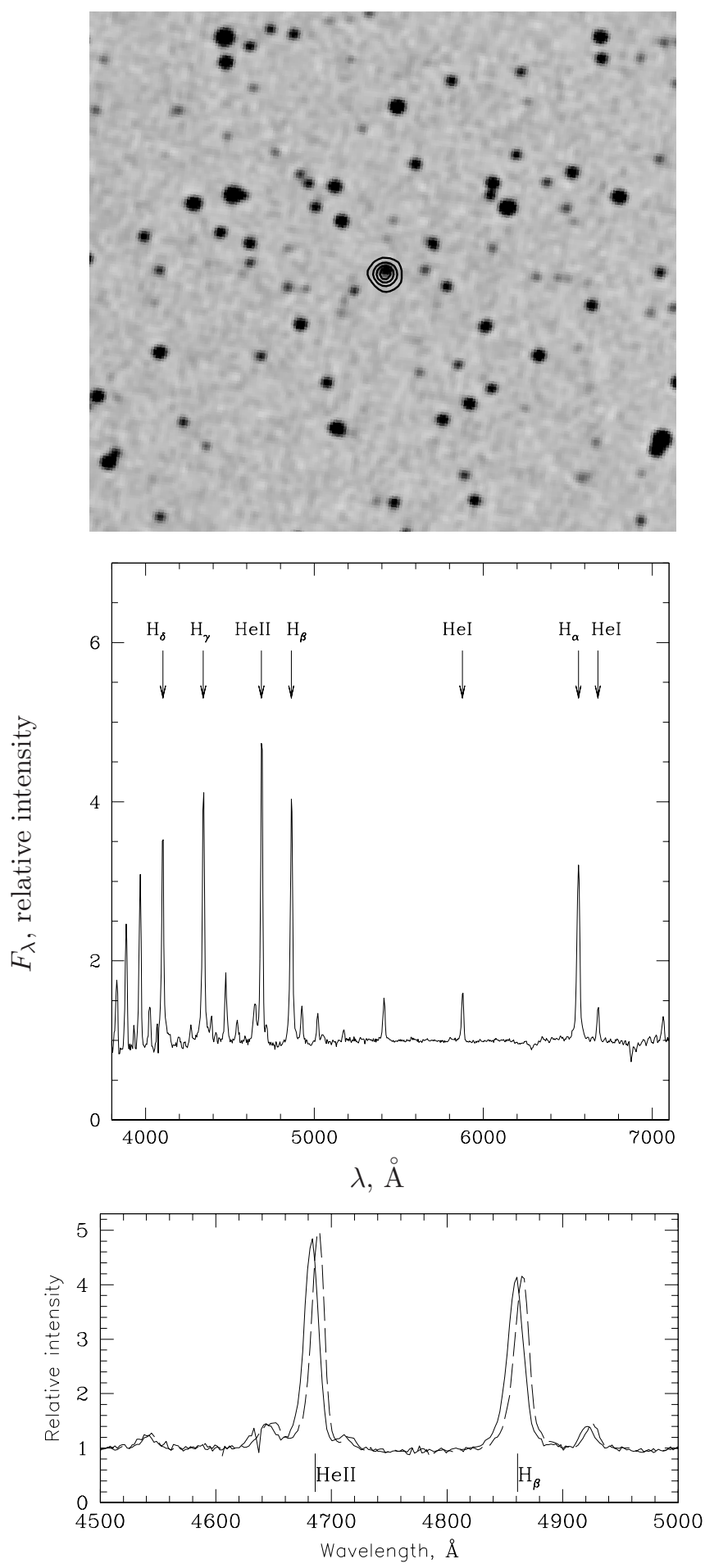

Fig. 5. (a) Image of the sky field around SWIFT J2341.0+ 7645 from digitized POSS plates; the contours indicate the soft X-ray intensity levels from XRT data. (b) The first of the two optical spectra for SWIFT J2341.0+7645 taken with the BTA telescope. (c) A magnified image of the spectral region in the wavelength range 4500 - $5000 \AA$. The solid line indicates the first spectrum of the source and the dashed line indicates the second spectrum taken $\approx 18$ min after the first one. A general shift of the second spectrum relative to the first one toward lower frequencies is clearly seen. 
$z=0)$ typical of the spectra of accreting white dwarfs (Fig 50 ). The emission line width $(20-25 \AA)$ clearly points to the line formation in an accretion disk around a compact object and corresponds to matter velocities in the disk $v \simeq 500-800 \mathrm{~km} / \mathrm{s}$, which are significantly higher than those in $S W I F T$ J2341.0+7645 but, nevertheless, are also observed in systems with white dwarfs (Howell et al. 2003). During one night, we took two spectra of the source with an interval of about $18 \mathrm{~min}$. We found that the positions of the emission lines in these spectra shifted by $4-5 \AA$, which exceeds considerably the positional error of the line centroids (Fig 5 c). A natural explanation of the observed effect is the orbital motion in the binary, but additional photometric and spectroscopic observations are required for firm conclusions to be reached and for the orbital period to be measured. It should be noted that the shift of various emission lines is inconsistent with the simple model in which all lines originate in the same part of the binary system. Such a behavior is not unusual, because different parts of the binary system (moving with different velocities around the system's center of mass) contain different sets of emission lines (see, e.g., the reviews on Doppler tomography of binary systems by Marsh and Horne (1988) and Steeghs et al. (1997)) .

Owing to the relatively long XRT observation, we managed to obtain a high-quality energy spectrum of the source (Fig (6). The spectrum can be satisfactorily described by a power law with a slope $\Gamma=0.71 \pm 0.08$ and a flux $F_{X}=(5.3 \pm 0.4) \times 10^{-12}$ erg $\mathrm{cm}^{-2} \mathrm{~s}^{-1}$ in the $2-10 \mathrm{keV}$ energy band. It is interesting to note that at energies $\sim 6.5 \mathrm{keV}$, there is evidence for the presence of a set of emission lines $(6.4,6.7,6.9 \mathrm{keV})$ typical of accreting white dwarfs. Adding a Gaussian at these energies to the model formally improves the quality of the fit to the spectrum but does not allow the line parameters to be properly constrained: the confidence level of the line is about $3 \sigma$ and its equivalent width is $\sim 0.8$ $\mathrm{keV}$.

In conclusion, note that fitting the spectrum by the model of partially absorbed bremsstrahlung with a temperature of $20 \mathrm{keV}$ (characteristic of white dwarfs in binary systems; see, e.g., Suleimanov et al. 2005) gives a poorer $\chi^{2}$ value, but the model parameters agree satisfactorily with those observed in similar systems: the absorption column density is $N_{H} \simeq 6 \times 10^{22}$ atoms $\mathrm{cm}^{-2}$ and the fraction of the absorbed emission in neutral matter is $C_{F} \simeq 0.73$ (Fig 6 , the dashed line).

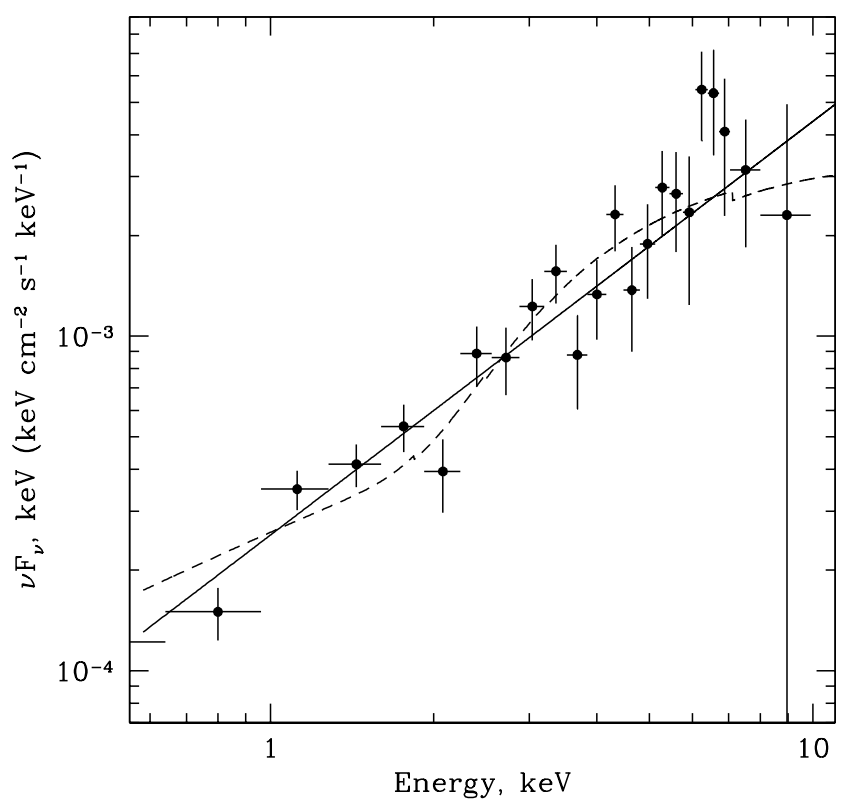

Fig. 6. XRT X-ray spectrum of SWIFT J2341.0+7645. The solid line indicates the best fit; the dashed line indicates the fit by the model of partially absorbed bremsstrahlung with a temperature of $20 \mathrm{keV}$.

Summarizing the aforesaid, we can assert that SWIFT J2341.0+7645 is a cataclysmic variable, an accreting white dwarf in a binary system.

\section{CONCLUSIONS}

We performed optical identifications of four hard X-ray sources from the Swift all-sky survey. Two sources were shown to be extragalactic in nature: SWIFT J0003.3+2737 is a Seyfert 2 galaxy at redshift $z=0.03946 \pm 0.00002$ and SWIFT $J 0113.8+2515$ is a blazar with a luminosity $L_{X} \simeq$ $1.9 \times 10^{46} \mathrm{erg} \mathrm{s}^{-1}$ at redshift $z=1.594$. The other two objects, SWIFT J2237.2+6324 and SWIFT $J 2341.0+7645$, belong to the class of cataclysmic variables (accreting white dwarfs) in our Galaxy. For SWIFT J2341.0+7645, we detected a spectral line shift during the observations, which is most likely due to the orbital motion in the binary system.

This study was supported by the Russian Foundation for Basic Research (project nos. 10-0200492, 10-02-01442, 10-02-00463，11-02-12285-ofim-2011, 11-02-12271-ofi-m-2011), the Presidium of Russian Academy of Sciences (the "Non-stationary 
phenomena in objects of the Universe" and "Active and Stochastic Processes in the Universe" Programs), the Program of the the President of the Russian Federation for support of scientific schools (grant NSh-5603.2012.2), grant no. MD1832.2011.2 from the President of the Russian Federation, grants from the Dynasty Foundation, and the State Contract no. 14.740.11.0611. We are grateful to S. Fabrika (Special Astrophysical Observatory, Russian Academy of Sciences) for his help in organizing the observations with the BTA telescope and for a discussion of the results.

\section{REFERENCES}

1. V. L. Afanasiev and A. V. Moiseev, Astron. Lett. 31, 194 (2005).

2. E. Barlow, C. Knigge, A. Bird, et al., Mon. Not. R. Astron. Soc. 372, 224 (2006).

3. W. Baumgartner, J. Tueller, C. Markwardt, G. Skinner, Bulletin of the American Astronomical Society, 41, 675 (2010)

4. I. Bikmaev, M. Revnivtsev, R. Burenin, and R. Syunyaev, Astron. Lett. 32, 588 (2006).

5. I. Bikmaev, R. Burenin, M. Revnivtsev, et al., Astron. Lett. 34, 653 (2008).

6. A. Bird, A. Bazzano, L. Bazzani, et al., Astrophys. J. Suppl. Ser. 186, 1 (2010).

7. R. Burenin, A. Meshcheryakov, M. Revnivtsev, et al., Astron. Lett. 34, 367 (2008).

8. R. Burenin, I. Bikmaev, M. Revnivtsev, et al., Astron. Lett. 35, 71 (2009).

9. G. Cusumano, V. La Parola, A. Segreto, et al., Astron. Astrophys. 524, 64 (2010).

10. J. Dickey and F. Lockman, Ann. Rev. Astron. Astrophys. 28, 215 (1990).

11. E. Filippova, S. Tsygankov, A. Lutovinov, and R. Syunyaev, Astron. Lett. 31, 729 (2005).

12. G. Fossati, L. Maraschi, A. Celotti, et al., Mon. Not. R. Astron. Soc. 299, 433 (1998).

13. N. Gehrels, G. Chinkarini, P. Giommi, et al., Astrophys. J. 611, 1005 (2004).

14. S. Howell, A. Adamson, and D. Steeghs, Astron. Astrophys. 399, 219 (2003).

15. R. Krivonos, M. Revnivtsev, A. Lutovinov, et al., Astron. Astrophys. 475, 775 (2007).

16. R. Krivonos, S. Tsygankov, M. Revnivtsev, et al., Astron. Astrophys. 523, A61 (2010).

17. A. Lutovinov, M. Revnivtsev, M. Gilfanov, et al., Astron. Astrophys. 444, 821 (2005).

18. A. Lutovinov and S. Tsygankov, Astron. Lett. 35, 433 (2009).

19. A. Lutovinov, R. Burenin, M. Revnivtsev, et al., Astron. Lett. 36, 904 (2010).
20. A. Lutovinov, R. Burenin, M. Revnivtsev, and I. Bikmaev, Astron. Lett. 38, 1 (2012).

21. T. Marsh and K. Horne, Mon. Not. R. Astron. Soc. 235, 269 (1988).

22. N. Masetti, R. Landi, M. Pretorius, et al., Astron. Astrophys. 470, 331 (2007).

23. N. Masetti, P. Parisi, E. Palazzi, et al., Astron. Astrophys. 519, 96 (2010).

24. M. Revnivtsev, A. Lutovinov, E. Churazov, et al., Astron. Astrophys. 491, 209 (2008).

25. S. Sazonov, E. Churazov, M. Revnivtsev M., et al., Astron. Astrophys. 444, L37 (2005)

26. S. Sazonov, M. Revnivtsev, R. Krivonos, et al., Astron. Astrophys. 462, 57 (2007).

27. S. Sazonov, R. Krivonos, M. Revnivtsev, et al., Astron. Astrophys. 482, 517 (2008)

28. D. J. Schlegel, D. P. Finkbeiner, and M. Davis, Astrophys. J. 500, 525 (1998).

29. D. Steeghs, E. Harlaftis, and K. Horne, Mon. Not. R. Astron. Soc. 290, L28 (1997).

30. V. Suleimanov, M. Revnivtsev, and H. Ritter, Astron. Astrophys. 435, 191 (2005).

31. P. Szkody, K. Nishikida, K. Long, and R. Fried, Astron. J. 121, 2761 (2001).

32. J. Tomsick, S. Chaty, J. Rodriguez, et al., Astrophys. J. 701, 811 (2009).

33. J. Tueller, W. Baumgartner, C. Markwardt, et al., Astrophys. J. Suppl. Ser. 186, 378 (2010).

34. R. E. Williams and D. H. Ferguson, Astrophys. J. 257, 672 (1982).

35. C. Winkler, T. Courvoisier, G. Di Cocco, et al., Astron. Astrophys. 411, L1 (2003). 